
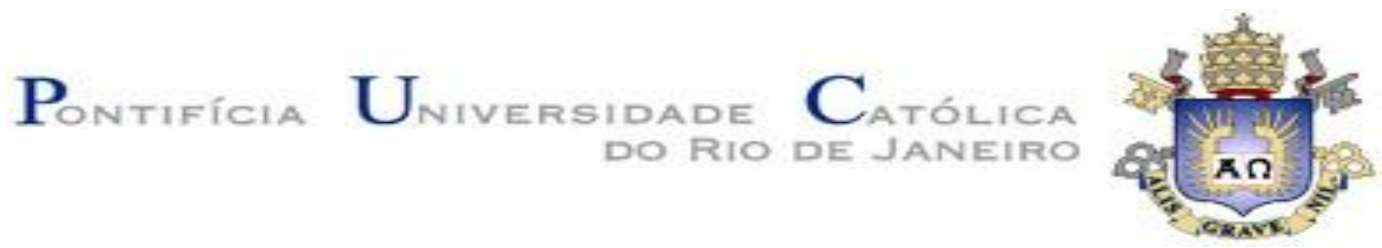

Ferry Sabel Belisario Benique

Carburização do DRI nas Zonas de Transição e Resfriamento de Reatores Tipo Midrex

Tese de Doutorado

Tese apresentada ao Programa de Pós-graduação em Engenharia de Materiais e de Processos Químicos e Metalúrgicos da Puc-Rio como requisito parcial para obtenção do título de Doutor em Engenharia de Materiais e de Processos Químicos e Metalúrgicos.

Orientador: Prof. José Carlos D’Abreu

Rio de Janeiro

Abril de 2011 

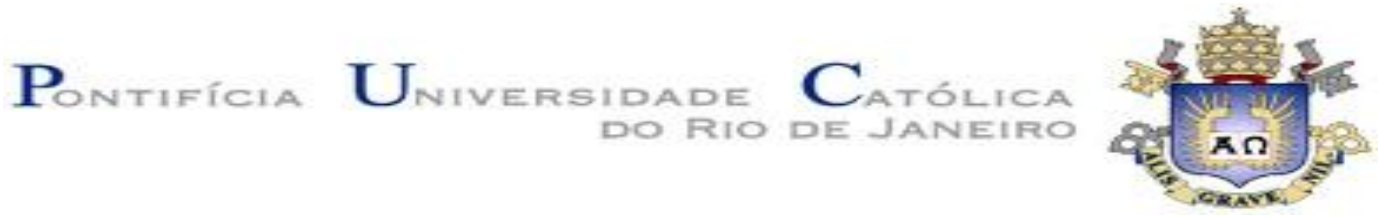

Ferry Sabel Belisario Benique

\section{Carburização do DRI nas Zonas de Transição e Resfriamento de Reatores Tipo Midrex}

Tese apresentada ao Programa de Pós-graduação em Engenharia de Materiais e de Processos Químicos e Metalúrgicos da Puc-Rio como requisito parcial para obtenção do título de Doutor em Engenharia de Materiais e de Processos Químicos e Metalúrgicos. Aprovada pela Comissão Examinadora abaixo assinada.

Prof. José Carlos D’Abreu

Orientador

Departamento de Engenharia de Materiais e de Processos Químicos e Metalúrgicos, PUC - Rio

Prof. Francisco José Moura Departamento de Engenharia de Materiais e de Processos Químicos e Metalúrgicos, PUC - Rio

Prof. Roberto José de Carvalho Departamento de Engenharia de Materiais e de Processos Químicos e Metalúrgicos, PUC - Rio

Prof. Cyro Takano USP - SP

Prof. Hélio Marques Kohler

Techn'os

Prof. José Eugenio Leal Coordenador Setorial do Centro Técnico Científico - PUC-Rio

Rio de Janeiro, 26 de Abril de 2011 
Todos os direitos reservados. É proibida a reprodução total ou parcial do trabalho sem autorização da universidade, do autor e do orientador.

Ferry Sabel Belisario Benique

Graduou-se em Engenharia Metalúrgica pela Universidade Nacional de San Agustín, Arequipa - Perú. Obteve o grau de Mestre em Engenharia Metalúrgica e de Materiais pela PUC-Rio em 2008.

Ficha Catalográfica

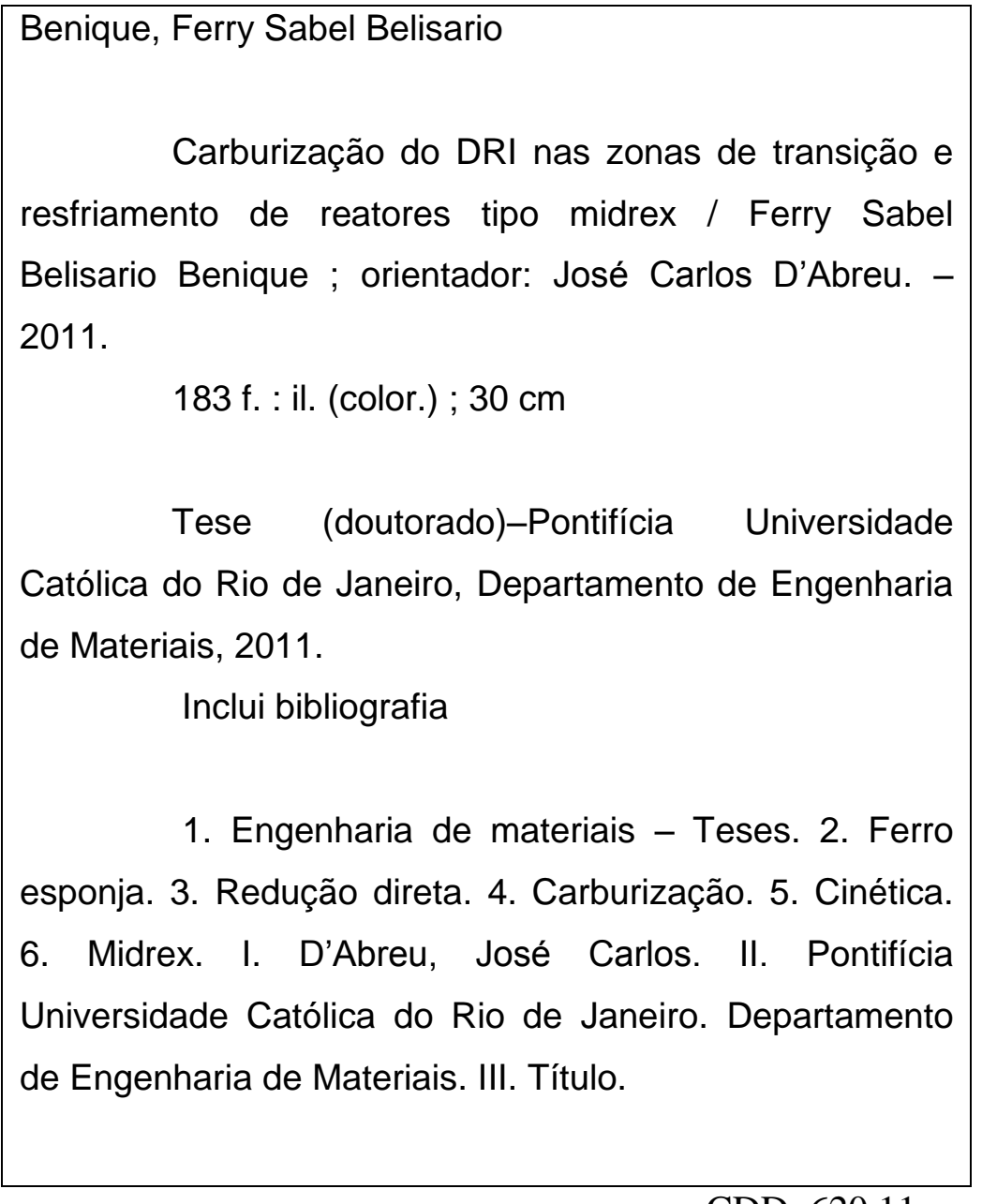

CDD: 620.11 
Agradeço a minha família, de forma especial, a minha querida Mãe por ter sido a minha grande incentivadora.

Agradeço a Deus por ser meu suporte em todos os momentos. 


\section{Agradecimentos}

Ao Prof. Dr. José Carlos D’Abreu pelo estímulo e orientação, fundamentais na realização deste trabalho, também, pela amizade, conversas e ensinamentos.

Ao Prof. Dr. Hélio Marques Kohler pelo apoio e orientações, pela sua amizade e companheirismo de sempre.

Agradeço à Samarco Mineração S/A por estimular a cooperação tecnológica e científica e pela oportunidade oferecida para participar das atividades industriais.

Agradeço a todos os profissionais da Samarco Mineração S/A, em especial aos das Gerencias Gerais de Marketing e Vendas, de Assistência Técnica e de Automação e Processo por ter viabilizado os ensaios metalúrgicos na usina e especialmente pela amizade oferecida.

Agradeço de forma especial ao meu caro amigo o Eng. Maurício Marcos Otaviano da Gerencia de Assistência Técnica da Samarco Mineração S/A por incentivar este trabalho, pelo valioso suporte técnico e pelas contribuições nas discussões sobre processos industriais. Agradeço também pela grande amizade construída, pelos conselhos oferecidos, pelo seu otimismo e bom humor. Grande abraço para toda a sua família, a sua esposa Ana, para a Marina e o Vitor !!!!.

Agradeço a toda a equipe dos laboratórios metalúrgico, químico, microscópico e físico da Samarco Mineração S/A pelo suporte técnico, prontidão e amizade oferecida ao longo do desenvolvimento experimental.

Ao Eng. Raimundo Nonato Rodrigues pelo companheirismo e amizade.

Ao CNPq e a PUC-Rio pelos auxílios concedidos no desenvolvimento deste trabalho.

A todos os professores do Departamento de Ciência dos Materiais e Metalurgia da PUC-Rio pelos ensinamentos e aos funcionários do DEMa que contribuíram 
direta ou indiretamente para a realização deste trabalho.

A minha família pelo apoio, incentivo e carinho em todo momento, de forma muito especial, a minha querida Mãe.

Aos meus amigos pela colaboração, amizade e sugestões dadas ao longo do trabalho.

A Deus pela minha saúde. 


\section{Resumo}

Belisario, Ferry Sabel Benique; D’Abreu, José Carlos. Carburização de DRI nas Zonas de Transição e Resfriamento de Reatores Tipo Midrex. Rio de Janeiro, 2011. 183p. Tese de Doutorado - Departamento de Engenharia de Materiais, Pontifícia Universidade Católica do Rio de Janeiro.

Nos últimos anos, esforços vêm sendo empreendidos no sentido de aumentar o conteúdo de carbono no ferro-esponja, com o intuito de utilizá-lo como fonte energética e como agente redutor dos óxidos residuais na operação dos fornos elétricos a arco - FEA. O presente trabalho, fruto de um projeto cooperativo da Samarco Mineração SA com o Grupo de Siderurgia da PUC-Rio, apresenta os resultados de experimentos realizados, nos laboratórios de ambas as Instituições simulando as condições operacionais de um forno Midrex. Para a simulação o reator foi dividido em três zonas: Redução, Transição e Resfriamento. Para cada uma delas foi concebido um conjunto de experimentos, estatisticamente planejados, de forma a permitir a mensuração cinética da carburização. Os experimentos envolvendo a redução e a carburização das pelotas de minério de ferro utilizaram similaridades fluidodinâmicas das escalas. Adicionalmente, um método para análise química dos teores de carbono grafítico e livre foi empregado com sucesso. Em seguida foram realizados os testes de carburização das zonas de transição e resfriamento e levantadas as curvas e equações cinéticas. Na zona de transição o agente carburizante foi o gás metano sendo a sua injeção feita nas temperaturas de $300,600,700$ e $850^{\circ} \mathrm{C}$. O modelo cinético utilizado foi uma equação linear de fluxo mássico. O valor da energia de ativação aparente encontrada para a carburização do DRI na zona de transição foi de $12,31 \mathrm{~kJ} / \mathrm{mol}$ indicando um controle cinético difusional. A carburização do DRI para esta região situou-se na faixa de $0.1 \% \mathrm{C}\left(300^{\circ} \mathrm{C}\right)$ a $3 \% \mathrm{C}\left(850^{\circ} \mathrm{C}\right)$, para um tempo de residência típico de 0,7 horas. Na zona de resfriamento o principal agente carburizante depende da temperatura: acima de $400^{\circ} \mathrm{C}$, foi o gás metano, enquanto nas temperaturas abaixo de $500^{\circ} \mathrm{C}$ foi o gás $\mathrm{CO}$. Nesta zona a carburização do DRI, nas temperaturas de 250 a $600^{\circ} \mathrm{C}$, foi suposta ser uma reação de primeira ordem em relação ao carbono. O resultado obtido para a energia de ativação aparente na zona de resfriamento foi $5,31 \mathrm{~kJ} / \mathrm{mol}$, consistente com um 
mecanismo controlado por difusão. Considerando os resultados experimentais obtidos, a carburização final do DRI nesta zona, tomando-se um tempo médio de residência de $\left(2,15 \mathrm{~h}\right.$ ) esteve na faixa de $2,0 \% \mathrm{C}$ ( periferia $/ 600^{\circ} \mathrm{C}$ ) a $3,0 \% \mathrm{C}$ centro $\left./ 250^{\circ} \mathrm{C}\right)$.

\section{Palavras-chave}

Ferro Esponja; Redução Direta; Carburização; Cinética; Midrex. 


\section{Abstract}

Belisario, Ferry Sabel Benique; D’Abreu, José Carlos (Advisor). DRI carburization in the Transition and Cooling Zones of Midrex Type Reactors. Rio de Janeiro, 2011. 183p, Doctoral Thesis - Departamento de Engenharia de Materiais, Pontifícia Universidade Católica do Rio de Janeiro.

In later years efforts are being made aiming at the increase in the carbon content in the DRI, this to enable it to act as energy source and reducing agent for the residual oxides in the operation of the electric arc furnaces - EAFs. The present work, product of a cooperative project between the Samarco Mining Co. and the Iron and Steelmaking Group from the University PUC-Rio, presents the obtained results for the experiments on DRI carburization, made at the laboratories of both institutions, under simulated conditions occurring in MIDREX reactors. To mimic the reactor operation, it was divided in three zones, quoting: Reduction, Transition and Cooling. For each of these zones a set of experiments were conceived and statistically planned to permit the carburization kinetic determination. It must be also added that fluidynamic scale similarity were respected in those same experiments. Further, a special chemical method was successfully employed to access the graphitic and free carbon DRI contents for the carburization tests performed for the Transition and Cooling zones. Based on the obtained results, sets of curves for carburization versus time were composed and kinetic equations, for various temperatures and gaseous atmospheres, were proposed. For the Transition zone the carburizing agent was the methane gas, being its injection made under the temperatures of 300, 600, 700 e $850^{\circ} \mathrm{C}$. The kinetic model utilized for the carburization in this zone was that of a mass flux linear equation. From this formalism, an apparent activation energy of $12.3 \mathrm{~kJ} / \mathrm{mol}$ was calculated indicating a difusional control. The total DRI carburization in this zone ranged from $0.1 \% \mathrm{C}\left(300^{\circ} \mathrm{C}\right)$ to $3 \% \mathrm{C}\left(850^{\circ} \mathrm{C}\right)$ for a typical residence time of 0.7 hours. In the Cooling Zone there was a temperature dependency ruling the carburizing agent role, above $400^{\circ} \mathrm{C}$ the gas methane is the main carburizing gas, but below $500^{\circ} \mathrm{C}$ the $\mathrm{CO}$ act as such. In this zone, the DRI 
carburization was fitted with a kinetic equation of the first order with respect to carbon. The obtained result for the apparent activation energy for this equation rated $5.31 \mathrm{~kJ} / \mathrm{mol}$, consistent with a difusional mechanism. Considering the experimental results for a residence time of 2.15 hours, the final carburization for the DRI in the Cooling Zone ranged from $2.0 \% \mathrm{C}$ ( furnace peripheral conditions / $600^{\circ} \mathrm{C}$ ) to $3.0 \% \mathrm{C}$ ( core conditions $/ 250^{\circ} \mathrm{C}$ ).

\section{Keywords}

Sponge Iron; Direct Reduction; Carburization; Kinetics; Midrex Process. 


\section{Sumário}

1 Introdução

2 Objetivos e relevância do trabalho

3 Considerações $\quad 28$

3.1. Considerações gerais 28

3.1.1. A cadeia produtiva da Siderurgia 30

3.1.2. Matérias primas empregadas na produção de aço líquido 32

3.1.3. Produção mundial de ferro esponja 32

3.1.4. Interesse na utilização de ferro esponja na fabricação de aço via FEA

3.1.5. Tecnologias de produção de DRI 40

3.1.6. Características físicas, químicas e metalúrgicas do DRI e HBI 44

3.1.7. Descrição do processo de redução direta Midrex 47

3.1.7.1. Zona de redução (ZR) 49

3.1.7.2. Zona de transição (ZT) 49

3.1.7.3. Zona de resfriamento ou arrefecimento (ZA) 49

3.1.8. Reformador do gás 51

3.1.9. Recuperação de calor 52

3.2. Considerações termodinâmicas da redução dos óxidos de ferro 54

3.2.1. Diagrama de equilíbrio $\mathrm{Fe}-\mathrm{O} 55$

3.2.2. Diagrama de equilíbrio ferro - carbono (Fe-C) 57

3.2.3. Redução dos óxidos de ferro pelo monóxido de carbono 58

3.2.3.1. Diagrama de oxi-redução -Diagrama de predominância Fe-O-C 58

3.2.3.2. Diagrama de Boudouard - A gaseificação do carbono 59

3.2.4. Redução dos óxidos de ferro pelo hidrogênio 60

3.2.4.1. Diagrama de predominância Fe-O-H 61

3.2.5. Redução dos óxidos de ferro pela mistura $\mathrm{CO}$ e $\mathrm{H}_{2}$

3.2.6. Termodinâmica da carburização 63

3.2.6.1. Termodinâmica da carburização na fabricação de carbeto de 
ferro

3.2.6.2. Termodinâmica da carburização na fabricação de DRI

3.2.6.3. Termodinâmica da deposição de carbono (carbono Pick Up) e a reação de Boudouard

3.3. Considerações cinéticas e fenomenológicas da redução dos óxidos de ferro

3.3.1. Cinética da redução de óxidos de ferro com $\mathrm{CO}$ puro

3.3.2. Cinética da redução de óxidos de ferro com a mistura $\mathrm{CO}-\mathrm{H}_{2} \quad 76$

3.3.3. Cinética da redução de óxidos de ferro com a mistura $\mathrm{CO}-\mathrm{H}_{2}$ contendo traços de enxofre

3.3.4. Cinética da redução de óxidos de ferro com a mistura $\mathrm{H}_{2}-\mathrm{CH}_{4}$

3.3.5. Mecanismos de reações de carburização e deposição de carbono

3.3.5.1. Cinética de reações de carburização e deposição de carbono em presença de ferro metálico

3.3.6. Mecanismos da redução de óxidos de ferro e da carburização de ferro esponja

3.3.6.1. Cinética da redução de óxidos de ferro e da carburização do ferro esponja nos processos de redução direta baseados em carvão 3.3.6.2. Cinética da redução de óxidos de ferro e da carburização do ferro esponja nos processos de redução direta baseados em gás : Midrex e HyL

3.3.7. Principais fatores que influenciam a carburização e a deposição de carbono no ferro esponja

3.3.7.1. Influência da temperatura e do tempo na carburização na deposição de carbono

3.3.7.2. Influência do ferro metálico e suas fases na carburização e na deposição de carbono

3.3.7.3. Influência da composição da mistura gasosa na carburização e na deposição de carbono

3.3.7.4. Influência da vazão da mistura gasosa na carburização e na deposição do carbono

3.3.7.5. Influência dos óxidos de ferro e da matéria prima na 
4 Modelamento cinético da zona de redução de reatores de cuba

4.1. Materiais utilizados na zona de redução (ZR)

4.2. Condições experimentais na zona de redução $(Z R)$

4.3. Resultados obtidos na zona de redução (ZR)

5 Desenvolvimento experimental

5.1. Experiências realizadas para o estudo da carburização na zona de transição $(\mathrm{ZT})$

5.1.1. Materiais utilizados na ZT

5.1.2. Equipamentos utilizados na ZT

5.1.3. Descrição do ciclo experimental na ZT

5.1.4. Planejamento experimental na $\mathrm{ZT}$

5.1.5. Condições experimentais na ZT

5.1.6. Desenho esquemático planejado para as experiências de carburização na ZT

5.2. Experiências realizadas para o estudo da carburização na zona de resfriamento $(Z A)$

5.2.1. Materiais utilizados na ZA

5.2.2. Equipamentos utilizados na ZA

5.2.3. Descrição do ciclo experimental na ZA

5.2.4. Planejamento experimental na ZA

5.2.4.1. Ensaios preliminares para a determinação da vazão crítica na ZT e ZA

5.2.5. Condições experimentais na ZA

6 Apresentação e discussão de resultados

6.1. Apresentação e discussão dos resultados obtidos na carburização na zona de transição (ZT)

6.1.1. Análise cinética na ZT

6.2. Apresentação e discussão de resultados obtidos na carburização do DRI na zona de arrefecimento (ZA) 
6.2.1. Análise termodinâmica na ZA

6.2.2. Determinação da vazão crítica para as experiências de carburização do DRI na ZA

6.2.3. Análise química das experiências de carburização do DRI na ZA152 6.2.4. Análise cinética das experiências de carburização do DRI na ZA 155 6.2.4.1. Modelamento cinético do teor de carbono total do DRI na ZA 156 6.2.4.2. Modelamento cinético do carbono cementítico do DRI na ZA 160 6.2.4.3. Carbono livre do DRI na ZA 164 6.2.4.4. Ferro metálico e o grau de metalização do DRI na ZA 166 6.2.4.5. Esquema da simulação global da carburização do DRI no forno de cuba

7 Conclusões

8 Sugestões para trabalhos futuros 173

9 Referências Bibliográficas 


\section{Lista de figuras}

Figura 1 - Produção mundial de aço (em milhões de toneladas) ao longo dos anos. ${ }^{(3)}$

Figura 2 - Produção mundial de aço por países em 2009 e 2010. ${ }^{(3)} \quad 29$

Figura 3 - Rotas tradicionais de produção de aço. ${ }^{(34,52)} 31$

Figura 4 - Evolução da produção de ferro esponja no mundo. ${ }^{(4)} 32$

Figura 5 - Produção mundial de DRI por região, em milhões de toneladas, ano 2008 e 2009. ${ }^{(4)}$

Figura 6 - Produção mundial de ferro esponja por processo, baseados em carvão e baseados em gás, em 2009. ${ }^{(4)}$

Figura 7 - Distribuição de custos variáveis na produção de aço líquido, 1999. ${ }^{(12)}$

Figura 8 - Consumo de energia de um FEA em função do grau de metalização do ferro esponja, para distintos percentuais de carregamento. ${ }^{(34)}$

Figura 9 - Efeito da temperatura do DRI e do teor de carbono do DRI no consumo de energia no FEA. ${ }^{(15)}$

Figura 10 - Influência do teor de carbono contido no pré-reduzido sobre os teores de ferro total e ferro metálico. ${ }^{(34)}$

Figura 11 - Evolução da produção de aço bruto via as rotas convencionais ${ }^{(19)}$.

Figura 12 - Áreas de operação, no diagrama Fe-C, dos processos de fabricação de ferro primário ${ }^{(19)}$.

Figura 13 - Fluxograma geral de produção de DRI / HBI mediante os processos de redução direta baseados em gás e em carvão. ${ }^{(17)}$

Figura 14 - Fluxograma de produção de ferro esponja no processo de redução direta baseado em gás, HYL III. ${ }^{(34)}$

Figura 15 - Fluxograma geral do processo de redução direta Midrex. ${ }^{(13)} 48$ Figura 16 - Fluxograma do processo Midrex sem recirculação gasosa 
na zona de resfriamento. ${ }^{(13)}$

Figura 17 - Reformador de gás natural utilizada pela tecnologia Midrex. (19)

Figura 18 - Catalisadores utilizados no processo de reforma do gás na tecnologia Midrex. ${ }^{(19)}$

Figura 19 - Diminuição de energia elétrica como função da temperatura de HDRI em diferentes relações de carga no FEA ${ }^{(22)}$.

Figura 20 - Diferentes opções de carregamento de HDRI para o FEA $^{(22)}$. 53

Figura 21 - Diagrama de equilíbrio ferro - oxigênio, (Fe-O). ${ }^{(38)} \quad 55$

Figura 22 - Diagrama de equilíbrio $\mathrm{Fe}-\mathrm{C}$. ${ }^{(38)}$

Figura 23 - Diagrama de Chaudron (Oxi-Redução). ${ }^{(38)} 59$

Figura 24 - Efeito da pressão sobre o equilíbrio da reação de

Boudouard. ${ }^{(40)}$

Figura 25 - Diagrama de predominância operacional Fe-O-H. ${ }^{(38,52,54)}$

Figura 26 - Diagrama de predominância operacional para o sistema

Fe-O-C-H. ${ }^{(38)}$

Figura 27 - Diagrama de estabilidade de fases para o sistema

Fe-C-H-O a $900 \mathrm{~K}$ e 1 atm de pressão total. Configuração da fase condensada metaestável. ${ }^{(55)}$

Figura 28 - Relação entre o conteúdo de carbono e a temperatura da carga no interior do forno de cuba. ${ }^{(58)}$

Figura 29 - Análise de raios $X$ em aglomerado de carbono. ${ }^{(58)}$

Figura 30 - Atividade calculada do carbono para o completo equilíbrio gasoso a 4 atm e temperaturas indicadas para o gás natural reformado contendo $73 \% \mathrm{H}_{2}, 18 \% \mathrm{CO}, 8 \% \mathrm{CO}_{2}$ e $1 \% \mathrm{CH}_{4}$ para os quais uma quantidade indicada de $\mathrm{H}_{2} \mathrm{O}$ é adicionada. ${ }^{(56)}$

Figura 31 - Curvas experimentais de TGA obtidas da redução de amostras grosseiras de óxido de ferro com CO puro a 800,850 e $900^{\circ} \mathrm{C}$. (37)

Figura 32 - Fração aparente de perda de peso de pelotas hematíticas comerciais de baixa sílica reduzidas com uma mistura gasosa contendo $75 \% \mathrm{H}_{2}$ e $25 \% \mathrm{CO}$ em função do tempo. ${ }^{(75)}$ 
Figura 33 - Fração aparente de perda de peso de pelotas hematiticas comerciais de baixa sílica reduzidas com uma mistura gasosa contendo $25 \% \mathrm{H}_{2}$ e $75 \% \mathrm{CO}$ em função do tempo. ${ }^{(75)}$

Figura 34 - Gráfico mostrando a variação do peso das pelotas com o tempo para várias condições. ( $R_{\text {red }}$ : taxa de redução, $R_{c}$ : taxa de deposição de carbono). ${ }^{(75)}$

Figura 35 - Padrões de difração de raios $X$ das amostras reduzidas. a) $1073 \mathrm{~K}$, b) $873 \mathrm{~K}^{(77)}$.

Figura 36 - Variação dos produtos da redução em função do tempo na temperatura de $1073 \mathrm{~K}^{(77)}$.

Figura 37 - Efeito da temperatura na redução de minério de ferro pela mistura gasosa contendo $25 \% \mathrm{H} 2-75 \%$ Ar. Vazão gasosa de 1 L/min. ${ }^{(94)}$

Figura 38 - Fração de cementita obtida em função da temperatura e do tempo de reação numa mistura gasosa contendo $35 \% \mathrm{CH}_{4}-55 \% \mathrm{H}_{2}-$ $10 \%$ Ar. $^{(94,95,96)}$

Figura 39 - Conteúdo de carbono total e carbono livre em amostra reduzida a $835^{\circ} \mathrm{C}$ em função do tempo de redução. ${ }^{(82)}$

Figura 40 - Variação do grau de metalização com o incremento do consumo na relação de carbono,em temperatura constante de $900^{\circ} \mathrm{C}^{(85)} .97$ Figura 41 - Redução de pelotas de hematita com $\mathrm{H}_{2}$, $\mathrm{CO}$ e gás Midrex a $850^{\circ} \mathrm{C}$ com uma vazão gasosa total de $2 \mathrm{~L} / \min ^{(89)}$.

Figura 42 - Desenho esquemático do reator de redução de minério de ferro mostrando as zonas de redução, de transição e resfriamento. ${ }^{(103)} 103$ Figura 43 - Geometria do forno de cuba. ${ }^{(104)}$ 104

Figura 44 - Efeito da temperatura sobre a taxa de deposição de carbono em presença de ferro metálico obtido previamente pela redução de minério hematítico granulado $\mathrm{com} \mathrm{H}_{2}$ em diferentes temperaturas $\left(400,600\right.$ e $\left.1000^{\circ} \mathrm{C}\right)$. As condições para deposição de carbono foram; a) 400 , b) 600 e c) $1000^{\circ} \mathrm{C}, 0.4 \mathrm{~atm} \mathrm{CO}$ (resto He) e com $\mathrm{W}_{\mathrm{Fe}}=660 \mathrm{mg} .{ }^{\left({ }^{(83)}\right.}$

Figura 45 - Efeito da temperatura relacionando a redução e a vazão gasosa para 30 minutos de redução. Símbolos fechados e símbolos 
abertos representam que houve ou não houve deposição de carbono, respectivamente. ${ }^{\left({ }^{87}\right)}$

Figura 46 - Efeito da quantidade do catalisador na deposição de carbono numa mistura gasosa contendo $50 \% \mathrm{H}_{2}$ e $50 \% \mathrm{CO}$ a $600^{\circ} \mathrm{C}$. O ferro foi previamente obtido pela redução de minério hematítico granulado. ${ }^{(83)}$

Figura 47 - Efeito da temperatura na taxa de deposição de carbono na presença de ferro poroso catalítico, pressões de 0.4 e 1 atm de CO. ${ }^{\left({ }^{83)}\right.} 110$ Figura 48 - Faixas de atividade catalítica do ferro com o teor de $\mathrm{H}_{2}$ na mistura gasosa. ${ }^{(98)}$

Figura 49 - Efeito da composição da mistura gasosa na deposição de carbono com misturas $\mathrm{H}_{2}-\mathrm{CO}$ a pressão atmosférica e $600^{\circ} \mathrm{C}$ na presença de $600 \mathrm{mg}$ de $\mathrm{Fe}$ (o Fe foi obtido previamente pela redução de minério hematítico granulado de $1 \mathrm{~mm}$ de diâmetro com $\left.\mathrm{H}_{2}\right) .{ }^{\left({ }^{(83)} \text {. }\right.}$ Línea tracejada representa resultados modelados.

Figura 50 - Efeito da composição gasosa $\mathrm{H}_{2}-\mathrm{CO}$ na taxa de deposição de carbono em $600 \mathrm{mg}$ de ferro poroso catalítico para as reações (55) e (56). ${ }^{(97)}$

Figura 51 - Efeito do $\mathrm{H}_{2} \mathrm{O}$ na taxa de deposição de carbono em $600 \mathrm{mg}$ de ferro poroso numa mistura de $\mathrm{CO}-\mathrm{H}_{2}-\mathrm{H}_{2} \mathrm{O}$ a pressão atmosférica. ${ }^{\left({ }^{(97)} 117\right.}$ Figura 52 - Efeito da vazão gasosa na redução em várias temperaturas, para 30 minutos de redução. Símbolos fechados e símbolos abertos representam que houve e não houve deposição de carbono, respectivamente. ${ }^{(87)}$

Figura 53 - Influencia típica da vazão gasosa na taxa de deposição de carbono em função da temperatura. ${ }^{(87)}$

Figura 54 - Variação da deposição de carbono em 1 atm de CO a $600^{\circ} \mathrm{C}$ com $600 \mathrm{mg}$ de $\mathrm{Fe}$ ( o Fe foi obtido previamente pela redução de hematita com $\mathrm{H}_{2}$ ) e com $600 \mathrm{mg}$ Fe eletrolítico. ${ }^{\left({ }^{83)}\right.}$

Figura 55 - Influência da temperatura no grau de redução de pelotas de minério de ferro sob condições de entrada na zona de redução do forno de cuba. ${ }^{(54)}$

Figura 56 - Influência da temperatura no grau de redução de pelotas 
de minério de ferro sob condições de gás de topo na zona de redução do forno de cuba. ${ }^{(54)}$

Figura 57 - Grau de redução em função do tempo para $900^{\circ} \mathrm{C}$ e um poder redutor de $0,85 .{ }^{(54)}$

Figura 58 - Grau de redução em função do tempo para $700^{\circ} \mathrm{C}$ e um poder redutor de $0,85 .{ }^{(54)}$. A linha pontilhada representa a remoção de oxigênio, a reta continua a carburização e a linha que se ajusta aos pontos experimentais a diferença.

Figura 59 - Deposição de carbono em função da temperatura e da vazão gasosa. ${ }^{(52)}$

Figura 60 - Etapas de pesquisa do programa cooperativo entre a PUCRio e a Samarco Mineração S/A sobre a carburização do DRI no forno de cuba tipo Midrex.

Figura 61 - Amostra de pelotas comerciais de minério de ferro tipo PDR-MX.

Figura 62 - Forno elétrico vertical.

Figura 63 - Retortas cilíndricas, externa e interna, utilizadas nas experiências de redução e carburização.

Figura 64 - Painel com rotâmetros controladores da vazão dos gases utilizados nas experiências de carburização.

Figura 65 - Desenho esquemático da aparelhagem montada para os experimentos de redução e carburização.

Figura 66 - Desenho esquemático com as condições experimentais para a carburização do DRI na zona de transição (ZT).

Figura 67 - Ciclos térmicos e temporais das experiências de carburização que simulam a periferia e o centro, nas três zonas do forno.

Figura 68 - Desenho esquemático planejado com as condições experimentais para a carburização do DRI nas três zonas do reator Midrex.

Figura 69 - Variação do carbono total no DRI em função da temperatura e do tempo para as condições experimentais na zona de transição. 
Figura 70 - Variação do coeficiente $C_{0}$ com o inverso da temperatura para as condições experimentais na zona de transição.

Figura 71 - Variação de energia livre de Gibb's com a temperatura das reações formadoras de carbono livre, considerando a composição do gás misturado na ZA.

$\mathrm{P}_{\mathrm{T}}=2.5 \mathrm{~atm}$.

Figura 72 - Variação de energia livre de Gibb's com a temperatura das reações formadoras de cementita, considerando a composição do gás misturado na $Z A$. $\mathrm{P}_{\mathrm{T}}=2.5 \mathrm{~atm}$.

Figura 73 - Vazão crítica na zona de transição (condições: periferia do forno).

Figura 74 - Vazão crítica na zona de arrefecimento (condições: centro do forno).

Figura 75 - Variação do teor de carbono total no DRI com o tempo para as condições de periferia da zona de resfriamento nas temperaturas de 250,500 e $600^{\circ} \mathrm{C}$.

Figura 76 - Variação do teor de carbono total no DRI com o tempo para as condições de centro da zona de resfriamento nas temperaturas de 250,500 e $600^{\circ} \mathrm{C}$.

Figura 77 - Variação de k com o inverso da temperatura, na modelagem cinética do teor de carbono total no DRI, para as condições de periferia e centro na zona de resfriamento do reator de cuba.

Figura 78 - Variação do teor de carbono cementítico no DRI com o tempo, para as condições de periferia da zona de resfriamento, nas temperaturas de 250,500 e $600^{\circ} \mathrm{C}$.

Figura 79 - Variação do teor de carbono cementítico no DRI com o tempo, para as condições de centro da zona de resfriamento, nas temperaturas de 250 a $600^{\circ} \mathrm{C}$.

Figura 80 - Variação de $\mathrm{k}$ com o inverso da temperatura, na modelagem cinética do teor de carbono cementítico no DRI, para as condições de periferia e centro na zona de resfriamento do reator de cuba.

Figura 81 - Variação do teor de carbono livre no DRI com o tempo, 
para as condições de periferia da zona de resfriamento nas

temperaturas de 250,500 e $600^{\circ} \mathrm{C}$.

Figura 82 - Variação do teor de carbono livre no DRI com o tempo, para as condições de centro da zona de resfriamento, nas temperaturas de 250,500 e $600^{\circ} \mathrm{C}$.

Figura 83 - Variação do ferro metálico em função do tempo, para as condições de periferia, na zona de resfriamento do reator de cuba. Figura 84 - Variação do ferro metálico em função do tempo, para as condições de centro, na zona de resfriamento do reator de cuba.

Figura 85 - Distribuição de freqüência em quartis do percentual de ferro metálico obtido na zona de arrefecimento do reator

Figura 86 - Perfil de carburização modelada para as três zonas do forno de cuba. 


\section{Lista de tabelas}

Tabela 1 - Países com maior produção de aço no mundo, em 2009 e 2010 (em milhões de toneladas). ${ }^{(3)}$

Tabela 2 - Evolução da produção mundial de DRI no mundo, em milhões de toneladas, (Mt), por processo. ${ }^{(4)}$

Tabela 3 - Tipos e classificação dos processos de redução direta ${ }^{(14)}$. 41

Tabela 4 - Características químicas do DRI / HBI dos diferentes processos de redução direta baseados nos tipos de redutores. ${ }^{(18)} 41$

Tabela 5 - Características físicas do DRI e HBI. ${ }^{(12,34)} 45$

Tabela 6 - Características químicas e metalúrgicas do DRI e HBI dos processos baseados em gás. ${ }^{(12,34)}$

Tabela 7 - Principais vantagens do HBI em comparação ao DRI. ${ }^{(12,34)} 46$

Tabela 8 - Relação H/C de algumas tecnologias de redução. ${ }^{(34,38)} 63$

Tabela 9 - Composição química da amostra utilizada nas experiências de carburização na zona de redução.

Tabela 10 - Condições experimentais na zona de redução (ZR).

Tabela 11 - Parâmetros cinéticos encontrados na carburização de ferro esponja na zona de redução de um forno de cuba. ${ }^{(52)}$

Tabela 12 - Composição química da amostra para o estudo da carburização na zona de transição.

Tabela 13 - Condições experimentais para a carburização na zona de transição (ZT).

Tabela 14 - Composição química da amostra para o estudo da carburização na zona de resfriamento.

Tabela 15 - Condições experimentais para a determinação da vazão crítica na zona de transição (ZA).

Tabela 16 - Condições experimentais para a determinação da vazão crítica na zona de arrefecimento (ZA).

Tabela 17 - Condições experimentais para a carburização na zona de resfriamento $(\mathrm{ZA})$.

Tabela 18 - Condições experimentais para determinar a carburização 
do DRI na periferia do forno Midrex.

Tabela 19 - Condições experimentais para determinar a carburização do DRI no centro do forno Midrex.

Tabela 20 - Análise química de carbono total nas pelotas após as experiências de carburização na zona de transição.

Tabela 21 - Resultados modelados para a carburização das pelotas após zona de transição.

Tabela 22 - Valores de Co observados e calculados para a zona de transição.

Tabela 23 - Parâmetros cinéticos obtidos no modelamento da carburização na zona de transição.

Tabela 24 - Energia livre de Gibb's das reações formadoras de carbono livre e cementita, considerando a composição do gás misturado na ZA.149 Tabela 25 - Energia livre de Gibb's das reações formadoras de carbono livre e cementita, considerando a composição do gás off take (gás de exaustão) na ZA.

Tabela 26 - Análise química do DRI obtido da ZA através das experiências de carburização que simulam a periferia do forno. Tabela 27 - Análise química do DRI obtido da ZA através das experiências de carburização que simulam o centro do forno. Tabela 28 - Resultados modelados para o carbono total do DRI, na periferia e no centro do forno, após zona de resfriamento.

Tabela 29 - Parâmetros cinéticos do teor de carbono total na carburização do DRI obtidos pelo modelo na zona de resfriamento.

Tabela 30 - Valores de k observados e cálculos para o modelamento do carbono total na zona de resfriamento.

Tabela 31 - Resultados modelados para o carbono cementítico do DRI, na periferia e no centro do forno, após zona de resfriamento.

Tabela 32 - Parâmetros cinéticos do teor de carbono cementítico na carburização do DRI obtidos pelo modelo na zona de arrefecimento. 162 Tabela 33 - Valores k observados e cálculos para o modelamento do carbono cementítico na zona de resfriamento.

Tabela 34 - Resultados experimentais do ferro metálico e metalização, 
para a periferia e para o centro do forno, na zona de resfriamento.

Tabela 35 - Resultados estatísticos da modelagem cinética dos

resultados experimentais do ferro metálico, obtidos nas experiências

de carburização na zona de arrefecimento do reator.

169 